\title{
Climate change brings the specter of new infectious diseases
}

\author{
Arturo Casadevall \\ Department of Molecular Microbiology and Immunology, Johns Hopkins School of Public Health, Baltimore, Maryland, USA.
}

limate change will bring major changes to the epidemiology of infectious diseases through changes in microbial and vector geographic range. Human defenses against microbial diseases rely on advanced immunity that includes innate and adaptive arms and endothermy, which creates a thermal restriction zone for many microbes. Given that microbes can adapt to higher temperatures, there is concern that global warming will select for microbes with higher heat tolerance that can defeat our endothermy defenses and bring new infectious disease.

\section{New microbial threats in a changing climate}

Almost three decades ago, experts began to sound the alarm that climate change could be associated with changes to the epidemiology of infectious diseases (1, 2). Since then a large body of literature has accumulated on this subject focused primarily on how climate will affect locations of pathogenic microbes and vectors of infectious diseases. In this Viewpoint, I focus on another threat: the strong possibility that new, previously unknown infectious diseases will emerge from warmer climates as microbes adapt to higher global temperatures that can defeat our endothermy thermal barrier.

The field of infectious diseases has the unenviable distinction of being the only subdiscipline of medicine that routinely has to deal with new diseases and where therapies lose their potency and value from drug resistance. New infectious diseases caused by pathogenic microbes not previously known to medicine emerge from acquisition from another species (e.g., species jump), as occurred with HIV, and from increased susceptibility in humans who become immunosuppressed, who are often the source of so-called opportunistic pathogens. The latter are rare and are usually the topic of case reports, although epidemics can occur, as exemplified by the outbreak of Exserohilum rostratum meningitis following the use of contaminated steroids in 2012 (3). Even though new infectious diseases are relatively frequent, with several reported each year, they involve a miniscule proportion of the microbial flora in the biosphere, which is a testament to remarkably powerful defense mechanisms in humans and mammals.

\section{Two major lines of defense}

The human organism is protected from infectious diseases by an advanced immune system that includes innate and adaptive arms and physical defenses. Although physical defenses such as skin are well known to physicians, the role of temperature is often unappreciated. Mammals are remarkable among animals in their endothermy and homeothermy, which maintains an elevated body temperature through life. Infectious diseases are acquired from other hosts (e.g., influenza virus) or directly from the environment, usually by inhalation (e.g., cryptococcosis and histoplasmosis). Microbes acquired from other humans are already adapted to mammalian temperatures, and these are not inhibited by endothermy. However, for many microbes, especially those in the environment that are adapted to cooler ambient temperatures, mammalian endothermy creates a thermal inhibitory zone. For example, about $95 \%$ of fungal species are inhibited by human core temperatures (4), which probably accounts for the paucity of fungal diseases in mammals relative to their high frequency in ectothermic species such as plants and insects.

Conflict of interest: The author has declared that no conflict of interest exists. 


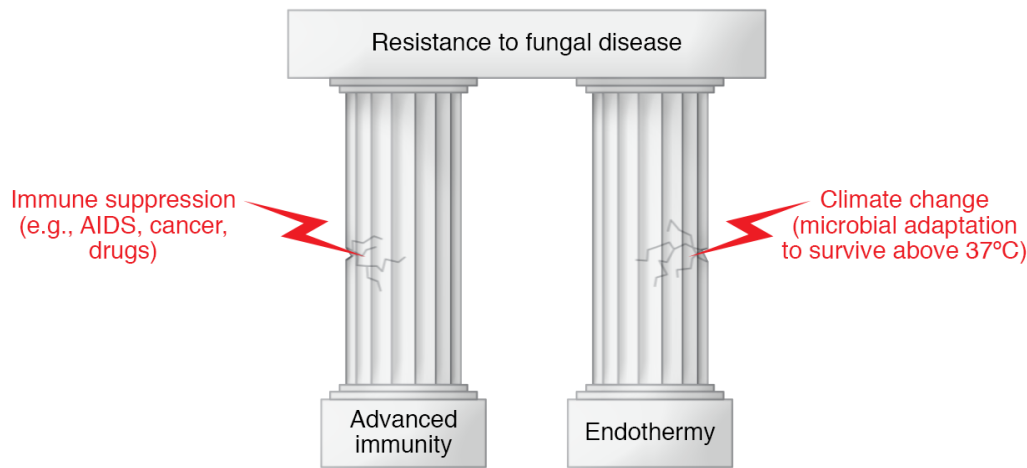

Figure 1. The remarkable resistance of humans (and mammals) to fungal diseases rests on the two pillars of advanced immunity and endothermy (i.e., the state of being warm-blooded). Immune suppression or climate change with microbial adaptation to survive at higher temperatures can undermine these pillars and make the host vulnerable to fungal disease.

of progressive warming, demonstrating that fungi are able to adapt rapidly to higher temperatures (9).

The concern is that there are a large number of fungal species that are pathogenic to plants and ectothermic animals, which means that they have the virulence tools to defeat immunity in these hosts, and if they adapt to higher temperatures, that could pose a new threat to humans. The same concern raised with the fungi applies to other microbes. Viruses are completely dependent on their hosts for replication and survival. As hosts adapt to higher temperatures, their associated viruses must follow suit to survive. Viruses associated with ectothermic animals that currently pose no threat to humans could jump to mammals if they acquire the capacity for replication at higher temperatures. Similar arguments apply to bacteria and parasites. Hence, the conceptual threat originally identified with fungi, and exemplified by $C$. auris as the canary in the coal mine, applies across the microbial world.

If these threats materialize, medicine will need to confront new infectious diseases for which it has no experience. Although the experience with Lyme disease, HIV, SARS coronaviruses, Zika virus, and $C$. auris shows that medicine and science can eventually respond successfully to previously unknown microbes with new diagnostics, control measures, and therapeutics, effective responses take time, and countless lives are lost in the meantime. However, various measures could be taken today to increase preparedness for confronting new infectious diseases.
Enhance surveillance for new diseases. Designing enhanced surveillance systems for human and animal diseases would provide early information about new pathogenic microbes. Early warning provides potentially actionable information that can be used to design containment measures and diagnostic tests and to develop new therapies and vaccines.

Promote research into nonhuman host-microbe interactions. New pathogenic microbes may use virulence strategies that are fundamentally different from those of known infectious diseases. For example, when HIV burst onto the scene in the 1980s, medical science had no experience with a virus whose pathogenic strategy crippled the immune system and left the host vulnerable to a variety of other lethal infectious diseases. Microbes that are currently pathogenic in plants and invertebrates may pose fundamentally different virulence strategies if they adapt to the human host. Today, medical research needs to be justified concerning its value for human health, and there is often little enthusiasm in diverting scarce research funds into nonhuman diseases. However, it is worth remembering that pioneering work into nonhuman retroviruses in the decades before the HIV epidemic created the knowledge base that allowed rapid progress in developing antiretroviral therapies once HIV was identified as a retrovirus.

Promote continued development of antimicrobial therapeutics. The majority of existing antimicrobial therapeutics target microorganisms associated with humans and livestock. However, these represent an infinitesimally small proportion of the microbial life in the biosphere, and there is no guarantee that currently available antimicrobial therapies would be effective against new pathogenic microbes. This is illustrated with $C$. auris, which is resistant to several of our most effective antifungal agents. Perhaps the greatest insurance against new organisms would be to develop host-targeted therapies that nonspecifically enhance the ability of hosts to resist infectious diseases.

Develop threat matrices to identify new likely potential threats. Threat matrices are already used to identify the most worrisome pathogenic microbes for biological warfare and bioterrorism (10), which guide research priorities and expenditures on preparedness measures, such as vaccines. Currently, there is probably not enough information available to construct such threat matrices, but any such exercise would inform on the research needed to obtain the required information. With enhanced information into the types of microbial diseases that affect plants and animals and the temperature tolerance of environmental microbes, it should be possible to develop threat matrices that stratify risk of new infectious diseases.

\section{Outlook}

Whereas climate change will almost certainly bring new infectious diseases and change the epidemiology of many existing diseases, it is worthwhile to note with optimism that humanity has confronted new deadly diseases in recent years, such as SARS coronaviruses and Ebola. Previous successes should not be a cause for complacency, and these reflect advances in science and clinical medicine that provide our species with tools to cope with new microbial threats. Clearly, prior success is no guarantee for future success, especially given that each new infectious disease brings in its own set of challenges. The best insurance against future threats is continued investment in surveillance, epidemiology, antimicrobial therapeutics, and basic research into mechanisms of microbial pathogenesis.

Address correspondence to: Arturo Casadevall, Department of Molecular Microbiology and Immunology, Johns Hopkins School of Public Health, 615 N. Wolfe Street, Room E5132, Baltimore, Maryland 21205, USA. Phone: 410.955.3457; Email: acasadevall@jhu.edu. 
1. Centers for Disease Control and Prevention. Emerging Infections: Microbial Threats to the United States. Washington, DC, USA: Institute of Medicine; 1992.

2. Patz JA, Epstein PR, Burke TA, Balbus JM. Global climate change and emerging infectious diseases. JAMA. 1996;275(3):217-223.

3. Andes D, Casadevall A. Insights into fungal pathogenesis from the iatrogenic epidemic of Exserohilum rostratum fungal meningitis. Fungal Genet Biol. 2013;61:143-145.

4. Robert VA, Casadevall A. Vertebrate endother- my restricts most fungi as potential pathogens. J Infect Dis. 2009;200(10):1623-1626.

5. Blehert DS, et al. Bat white-nose syndrome: an emerging fungal pathogen? Science. 2009;323(5911):227.

6. Garcia-Solache MA, Casadevall A. Global warming will bring new fungal diseases for mammals. MBio. 2010;1(1):e00061-10.

7. Lockhart SR, et al. Simultaneous emergence of multidrug-resistant Candida auris on 3 continents confirmed by whole-genome sequencing and epidemiological analyses. Clin Infect Dis.
2017;64(2):134-140.

8. Casadevall A, Kontoyiannis DP, Robert V. On the emergence of Candida auris: climate change, azoles, swamps, and birds. MBio. 2019;10(4):e01397-19.

9. de Crecy E, Jaronski S, Lyons B, Lyons TJ, Keyhani NO. Directed evolution of a filamentous fungus for thermotolerance. BMC Biotechnol. 2009;9:74

10. Rotz LD, Khan AS, Lillibridge SR, Ostroff SM, Hughes JM. Public health assessment of potential biological terrorism agents. Emerging Infect Dis. 2002;8(2):225-230. 